\title{
Aplicação do instrumento LASSI e ILS em estudantes de farmácia para caracterização de ensino-aprendizagem
}

Application of the LASSI and ILS instrument in pharmacy students for characterization of teaching-learning

Aplicación del instrumento LASSI e ILS en estudiantes de farmacia para la caracterización de la enseñanza-aprendizaje Universidade Federal de Sergipe, Brasil

Wellington Barros da Silva

\begin{abstract}
Resumo
O conceito de estilo na linguagem pedagógica é frequentemente utilizado para abordar os diferentes tipos de comportamentos que estão reunidos em um único estereótipo, o que de certa forma, se torna útil para a classificação e análises comportamentais. Desta forma, o trabalho teve como objetivo caracterizar os estilos e estratégias de aprendizagem entre estudantes universitários do curso de graduação em Farmácia. O trabalho foi realizado em dois momentos: Primeiramente foi realizado a fundamentação teórica sobre o processo de ensino-aprendizagem; Logo após, foi realizada a caracterização das estratégias de aprendizagem utilizando o questionário LASSI (Learning and Study Strategies Inventory) e para a caracterização dos estilos de aprendizagem, o instrumento ILS (Index of. Learning Styles). Os resultados apresentados não demostraram relações de ensino-aprendizagem durante o tempo de graduação em relação as estratégias de aprendizagem. Enquanto, os estilos de aprendizagem, mais observadas, foram os estilos sensorial/intuitivo. Além disso, existiu uma predominância entre os estudantes dos estilos sensorial, visual e sequencial, tendo como o estilo menos frequente entre os estudantes, o intuitivo. Diante os dados apresentados, podese observar à escassez de estudos que avaliaram os estilos ou as estratégias de aprendizagem entre estudantes universitários brasileiros. Sendo assim, se torna necessário mais investigações no âmbito da farmácia para a verificação de mais detalhes sobre fatores e suas relações com a utilização desses estilos e estratégias de aprendizagem no incremento acadêmico dos estudantes.
\end{abstract}

Palavras-chave: Educação em farmácia; Ensino; Método de ensino. 


\begin{abstract}
The concept of style in pedagogical language is often used to address the different types of behaviors that are united in a single stereotype, which somehow becomes useful for classification and behavioral analysis. Thus, the work aimed to characterize the styles and learning strategies among university students of the undergraduate course in Pharmacy. The work was carried out in two stages: First, the theoretical foundation on the teaching-learning process was carried out; Afterwards, the characterization of the learning strategies was carried out using the LASSI (Learning and Study Strategies Inventory) questionnaire and, for the characterization of the learning styles, the ILS instrument (Index of Learning Styles). The results presented did not demonstrate teaching-learning relationships during graduation time in relation to learning strategies. While, the most observed learning styles were the sensory/intuitive styles. In addition, there was a predominance among students of sensory, visual and sequential styles, with the less frequent style among students, the intuitive. In view of the data presented, one can observe the scarcity of studies that assessed learning styles or strategies among Brazilian university students. Therefore, further investigations are needed in the field of pharmacy to verify more details about factors and their relationships with the use of these learning styles and strategies in the academic growth of students.
\end{abstract}

Keywords: Pharmacy education; Teaching; Teaching method.

\title{
Resumen
}

El concepto de estilo en el lenguaje pedagógico se utiliza a menudo para abordar los diferentes tipos de comportamientos que se unen en un solo estereotipo, que de alguna manera se vuelve útil para la clasificación y el análisis del comportamiento. Así, el trabajo tuvo como objetivo caracterizar los estilos y estrategias de aprendizaje entre los estudiantes universitarios de la carrera de Licenciatura en Farmacia. El trabajo se llevó a cabo en dos etapas: en primer lugar, se realizó el fundamento teórico sobre el proceso de enseñanza-aprendizaje; Posteriormente, se realizó la caracterización de las estrategias de aprendizaje mediante el cuestionario LASSI (Learning and Study Strategies Inventory) y, para la caracterización de los estilos de aprendizaje, el instrumento ILS (Index of Learning Styles). Los resultados presentados no demostraron relaciones enseñanza-aprendizaje durante el tiempo de graduación en relación a las estrategias de aprendizaje. Mientras, los estilos de aprendizaje más observados fueron los estilos sensoriales / intuitivos. Además, hubo predominio entre los estudiantes de los estilos sensorial, visual y secuencial, siendo el estilo menos frecuente entre los estudiantes, el intuitivo. A la vista de los datos presentados, se puede observar la escasez de estudios que evaluaran estilos o estrategias de aprendizaje entre los estudiantes universitarios brasileños. Por lo tanto, se necesitan más investigaciones en el campo de la farmacia para verificar más detalles sobre los factores y sus relaciones con el uso de estos estilos y estrategias de aprendizaje en el crecimiento académico de los estudiantes.

Palabras clave: Educación en farmacia; Enseñanza; Método de enseñanza.

\section{Introdução}

O conceito de estilo na linguagem pedagógica é frequentemente utilizado para abordar os diferentes tipos de comportamentos que estão reunidos em um único estereótipo, o que de certa forma, se torna útil para a classificação e análises comportamentais (Czepula et al, 2016). Em uma tentativa de compreender e atender às necessidades individuais dos alunos, os educadores propuseram a existência de estilos cognitivos e de aprendizagem. O estilo de aprendizagem é um termo usado para descrever as diferentes formas e preferências utilizadas pelas pessoas para aprender e são essas peculiaridades que contribui de forma individual para o desenvolvimento do aprendizado (Nisly et al, 2020).

A avaliação de resultados de aprendizagem é importante para que exista um processo onde os alunos possam monitorar e controlar sua cognição, estados afetivos e comportamentais (Calthorpe et al, 2019). Neste contexto, o conhecimento e consciência do estilo de aprendizagem do aluno é importante para os processos de ensino e aprendizagem. Este conhecimento pode ser usado para desenvolver o programa educacional e melhorar a aprendizagem do aluno (McKiernan et al, 2020).

A metodologia de aprendizagem utilizada e os seus perfis de motivação estão intimamente correlacionados. Desta forma, a compreensão das estruturas de aprendizagem pelos discentes, torna possível manter a motivação dos alunos por um tempo mais longo, o que acarreta na formação continua do profissional, mesmo após seu desligamento com a unidade formadora (Yamamura et al, 2018). Os pesquisadores em educação farmacêutica e médica estão cada vez mais conscientes que o uso de uma variedade de métodos de ensino pode melhorar a retenção do conteúdo, além de desenvolver a capacidade de resolver problemas diante determinadas situações. Diversas pesquisas sugerem que a compreensão do estilo de aprendizagem 
que o aluno está propenso é útil para fornecer uma experiência de aprendizagem bem-sucedida não importando o método de ensino (Childs-Kean et al, 2020).

Czepula e colaboradores (2016) relatam que os alunos estão conscientes das suas próprias preferências de aprendizagem e usam métodos de aprendizagem que é mais conveniente para eles, particularmente em situações no qual o estilo de ensino do instrutor não corresponde ao estilo de aprendizagem preferido do estudante. Os professores também podem se beneficiar de uma maior compreensão dos estilos de aprendizagem dos estudantes como um meio para adaptar aos seus estilos de ensino para melhor atender às necessidades dos alunos.

Diante do exposto, este artigo busca investigar os estilos e as estratégias de aprendizagem entre estudantes do curso de Farmácia, visando analisar as relações entre os tipos de aprendizagem dos estudantes e o seu desempenho acadêmico, motivação e preferências.

\section{Metodologia}

A pesquisa empírica trata-se de um estudo de Caso, observacional, descritivo com delineamento transversal e foi realizada com alunos do curso de graduação em Farmácia da Universidade Federal de Sergipe (Campus de São Cristóvão).

Os sujeitos da pesquisa, que constituem a amostra do estudo, são os alunos que faziam parte da população-elegível, presentes em sala de aula no momento da aplicação do instrumento de coleta de dados e que concordaram com a sua realização por meio da assinatura do Termo de Consentimento Livre e Esclarecido (TCLE). A presente pesquisa foi aprovada no Comitê de Ética e Pesquisa Envolvendo Seres Humanos da Universidade Federal de Sergipe CEP/UFS N ${ }^{\circ}$ CAAE - 0050.0.107.000-11.

Os instrumentos utilizados para a caracterização foram: o Índice dos Estilos de Aprendizagem (Index of Learning Styles - ILS e o Inventário de Estudos e Estratégias de Aprendizagem (Learning and Study Strategies Inventory - LASSI).

A coleta dos dados foi realizada em sala de aula, sempre antecedendo o início das aulas e com consentimento do professor. Foram esclarecidos os objetivos da pesquisa e após preenchimento do TCLE pelo aluno, os instrumentos eram aplicados.

Variáveis de análise:

- $\quad$ Sociodemográficas: idade, gênero, trabalha em alguma atividade remunerada;

- $\quad$ Socioeducacionais: instituição onde cursou o ensino médio (pública ou privada), período letivo;

- $\quad$ Estilos de aprendizagem (ILS);

- $\quad$ Estratégias de estudo e aprendizagem (LASSI).

Para a caracterização das estratégias de aprendizagem, utilizou-se a versão brasileira do LASSI validada por Bartalo (2006).

\section{Resultados e Discussão}

\section{Caracterização das estratégias de aprendizagem (LASSI)}

Esta seção apresenta e discute os resultados da caracterização dos estilos de aprendizagem a partir dos dados obtidos pela aplicação do instrumento LASSI. Descrevem-se os dados sociodemográficos e socioeducacionais dos estudantes, os escores médios e as pontuações gerais obtidas para cada subescala das estratégias de aprendizagem estudadas.

\section{Dados sociodemográficos e socioeducacionais}

No inventário LASSI participaram 192 alunos com uma média de idade de 21,3 $( \pm 3,12)$ (mediana $=21$ anos). Desses alunos $143(74,5 \%)$ eram do gênero feminino e 49 (25,5\%) do gênero masculino; 111 (57,8\%) estudaram o ensino médio em escola particular e 81 (42,2\%) em escola pública. 
Para o desenvolvimento da pesquisa foi identificada e selecionada aleatoriamente uma turma/disciplina por período acadêmico com o intuito de facilitar a aplicação do instrumento da pesquisa; deste modo cada período correspondeu às seguintes turmas/disciplinas: $1^{\circ}$. Período: Introdução à Farmácia (carga horária: 02 h); $2^{\circ}$. Período: Biofísica (carga horária: 06 h); $3^{\circ}$. Período: Assistência Farmacêutica (carga horária: 04 h); 4․ Período: Deontologia e Legislação Farmacêutica (carga horária: 02 h); $5^{\circ}$. Período: Farmacologia (carga horária: 04 h); $8^{\circ}$. Período: Microbiologia Clínica (carga horária: 04 h) e $9^{\circ}$ Período: Atenção Farmacêutica (carga horária: 04 h).

Foi analisado que do quarto período ao primeiro houve um aumento significativo do ingresso no curso de alunos oriundos de escola pública, esse fato pode ser atribuído ao sistema de cotas que entrou em vigor na UFS a partir do período 2010.1, ano em que também passou a entrar uma turma de quarenta alunos a cada semestre.

Entre os estudantes de Farmácia que participaram da pesquisa, 68 (35,4\%) responderam que exercem alguma atividade remunerada. Este percentual parece aumentar entre aqueles estudantes que se encontram nos períodos finais do curso $(52,9 \%)$ quando comparado aos períodos iniciais $(20 \%$ e $16 \%)$.

\section{Estratégias de aprendizagem}

Destacam-se como estratégias mais utilizadas pelos estudantes aqueles referentes a preocupações ao estudar $(4,22 \pm$ 0,992), processamento da informação $(3,84 \pm 1,11)$ e Auxiliares de estudo $(3,34 \pm 1,512)$, os quais representam as estratégias de utilização de técnicas ou métodos próprios que auxiliam os estudantes no seu processo de aprendizagem.

De uma forma geral observam-se problemas com as estratégias relacionadas com os níveis de concentração e motivação para a aprendizagem, apesar de os estudantes demonstrarem preocupação com os estudos. Os resultados encontramse descritos na Tabela 1.

Tabela 1. Estratégias de aprendizagem entre os estudantes do Curso de Farmácia da UFS, Campus de São Cristóvão/SE.

\begin{tabular}{|l|l|l|}
\hline Estratégias de aprendizagem & Média & Desvio padrão (DP) \\
\hline Processamento da informação & $\mathbf{3 . 8 4}$ & 1.11 \\
\hline Ansiedade & 2.58 & 1.267 \\
\hline Organização do tempo & 2.77 & 1.265 \\
\hline Concentração & 2.20 & 1.155 \\
\hline Atitude & 1.60 & 1.003 \\
\hline Preocupações ao estudar & $\mathbf{4 . 2 2}$ & 0.992 \\
\hline Seleção de ideias principais & 2.40 & 1.191 \\
\hline Auxiliares de estudos & $\mathbf{3 . 3 4}$ & 1.512 \\
\hline Motivação & 2.79 & 1.435 \\
\hline Internet & 2.96 & 1.58 \\
\hline
\end{tabular}

Fonte: Autores.

A Figura 1 mostra que a estratégia para a qual foi observada a menor pontuação entre os estudantes foi atitude $(1,60 \pm$ 1,003). Está junto com as estratégias de motivação (que no presente estudo também obteve baixos escores) expressa o nível de importância e interesse atribuídos pelos estudantes às atividades de ensino e ao desempenho acadêmico. Os estudantes também obtiveram baixos escores na utilização de estratégias de concentração $(2,20 \pm 1,155)$ e na seleção de ideias principais $(2,40 \pm$ 1,191), o que evidencia nos alunos dificuldades para identificar e avaliar a relevância das informações e, portanto, demonstram problemas de foco no processo de aprendizagem. 
Figura 1. Estratégias de aprendizagem dos estudantes do Curso de Farmácia da UFS (n= 192), Campus São de Cristovão/SE.

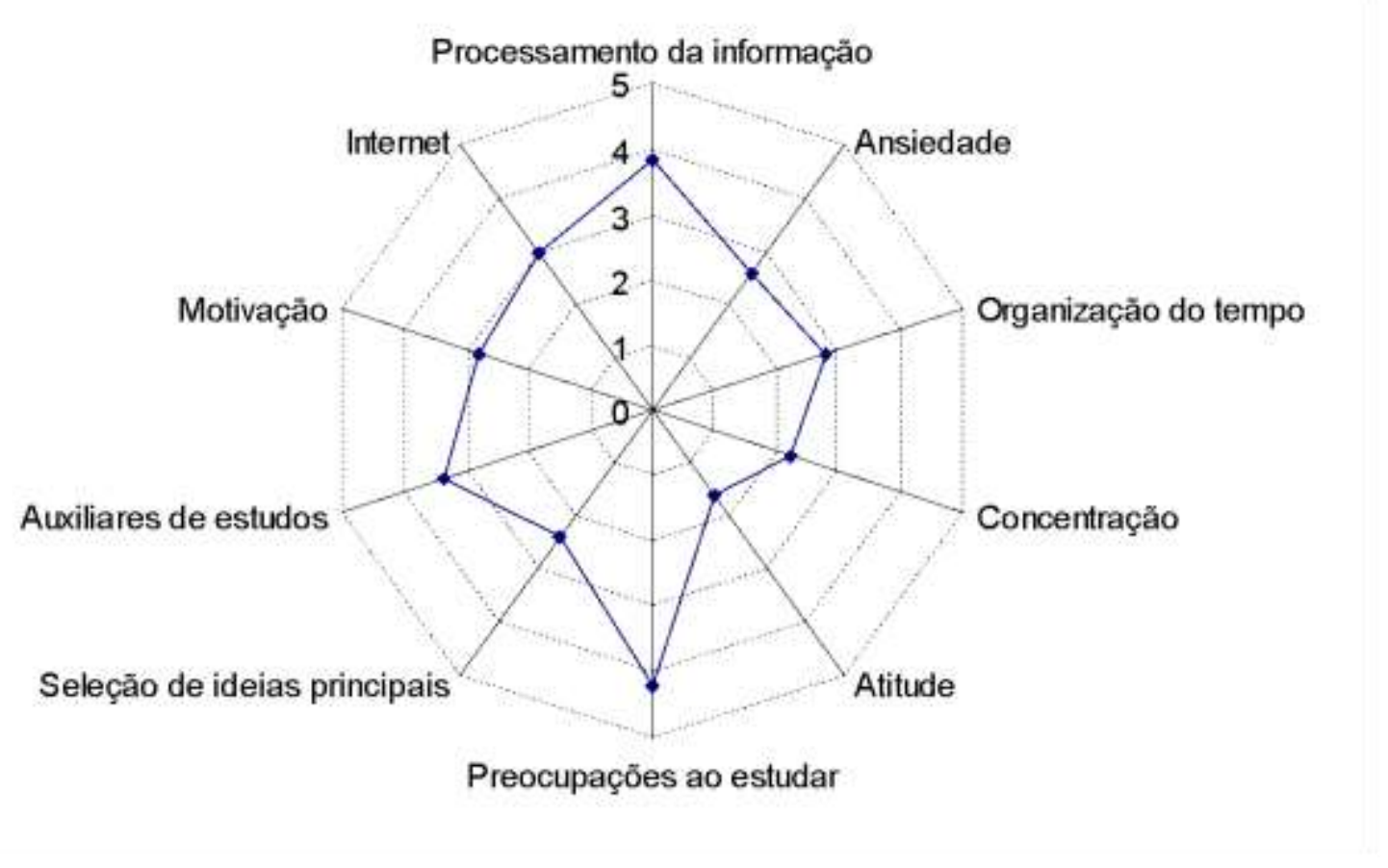

Fonte: Autores.

Em relação às variáveis independentes utilizou-se o teste $\mathrm{t}$ (bicaudal) para comparar os escores médios observados entre os gêneros (masculino e feminino), entre os estudantes segundo a sua procedência no ensino médio (se estudou em escola pública ou privada) e finalmente entre os estudantes que exerciam atividade remunerada ou não. Não foram verificadas diferenças estatisticamente significativas entre os escores desses grupos.

\section{Estilos de aprendizagem}

As preferências dos alunos referentes aos estilos de aprendizagem foram caracterizadas de acordo com as quatro dimensões: Percepção (estilos sensorial e intuitivo), Retenção (estilos visual e verbal), Processamento (estilos ativo e reflexivo) e Compreensão (estilos sequencial e global). Os resultados encontram-se descritos na tabela 2.

Tabela 2. Estilos de aprendizagem dos estudantes do Curso de Farmácia da UFS, Campus de São Cristóvão/SE. Modelo ILS (Felder-Silverman, 2005).

\begin{tabular}{l|l|l|l}
\hline Dimensão & Estilo & Frequência (\%) & Média (escala ILS) (EP) \\
\hline \multirow{2}{*}{ Percepção } & Sensorial & $151(87,8)$ & $5,3(0,22)$ \\
\cline { 2 - 4 } & Intuitivo & $15(8,7)$ & $1,9(0,27)$ \\
\hline \multirow{2}{*}{ Retenção } & Visual & $120(69,8)$ & $4,9(0,25)$ \\
\cline { 2 - 4 } & Verbal & $46(26,7)$ & $3,2(0,34)$ \\
\hline \multirow{2}{*}{ Processamento } & Ativo & $97(56,4)$ & $3,4(0,23)$ \\
\cline { 2 - 4 } & Reflexivo & $69(40,1)$ & $3,0(0,27)$ \\
\hline \multirow{2}{*}{ Compreensão } & Sequencial & $106(61,6)$ & $3,4(0,22)$ \\
\cline { 2 - 4 } & Global & $59(34,3)$ & $2,7(0,28)$ \\
\hline
\end{tabular}

Fonte: Autores. 
A Figura 2 mostra de forma geral a prevalência nos estilos sensorial, visual e sequencial de aprendizagem dos alunos do curso de Farmácia da UFS. Estes dados são congruentes com os encontrados por Teevan e colaboradores (2011), que também detectou entre alunos de Farmácia preferência moderados para os estilos de aprendizagem, sensorial, visual e sequencial. Neste estudo os estudantes americanos não demonstraram preferência pelo estilo ativo ou reflexivo.

Figura 2. Estilos de aprendizagem dos estudantes do curso de Farmácia da UFS (n=171), Campus de São Cristovão/SE.

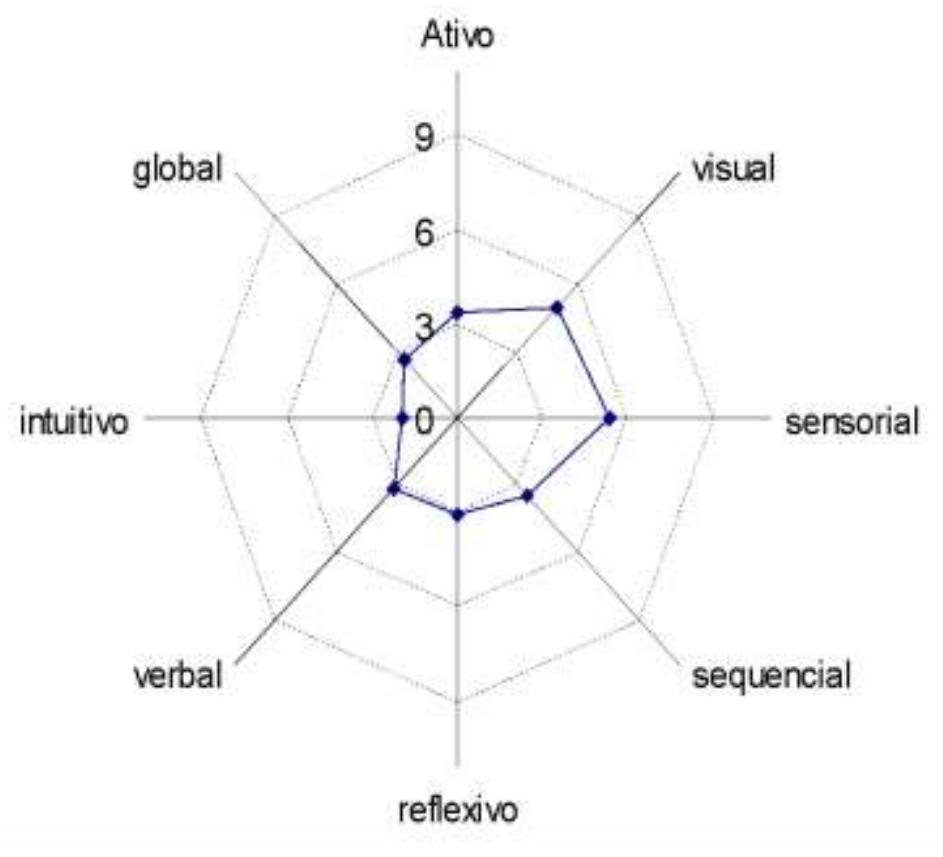

Fonte: Autores.

Houve predominância entre os estudantes dos estilos sensorial (87,8\%), visual $(69,8 \%)$ e sequencial $(61,6 \%)$, sendo que o estilo intuitivo foi o menos frequente entre os estudantes com 8,7\%. A maior diferença entre estilos foi observada entre os estilos sensorial e intuitivo, enquanto a menor diferença entre os estudantes ocorreu nas preferências de processamento da informação: ativo $(56,4 \%)$ e reflexivo $(40,1 \%)$.

Em relação aos escores, as maiores médias foram observadas para os estilos sensorial/intuitivo, com média 5,02 $( \pm 2,794)$ e estilos visual/verbal, com média $4,40( \pm 2,763)$.

A caracterização dos estilos de aprendizagem predominantes entre os alunos do curso de Farmácia da UFS, de forma geral, apresenta algumas incongruências com o perfil de egresso preconizado pelas DCNs. As DCNs recomendam expressamente um perfil de egresso/profissional “(...) com formação generalista, humanista, crítica e reflexiva, para atuar em todos os níveis de atenção à saúde, com base no rigor científico e intelectual” (Brasil, 2002). Os baixos escores nos estilos global, verbal, intuitivo e ativo/reflexivo podem vir a comprometer algumas das competências e habilidades gerais necessárias para este perfil, como liderança, comunicação, administração e gerenciamento e educação permanente.

Na pesquisa realizada por Barreto et al. (2019) em estudantes de medicina em Belém - PA, foi verificado que a correlação positiva que existe entre estratégias de planejamento, organização e outros tipos de domínios podem revelar que o planejamento do estudo está diretamente associado às melhores estratégias de aprendizagem e que juntamente com busca por fontes de melhor qualidade técnica, resulta em uma maior retenção do conhecimento pelo aluno.

Não houve diferença estatisticamente significativa de gênero quanto aos estilos de aprendizagem. Este resultado contrasta com outros estudos com estudantes brasileiros que verificaram a influência do gênero nos estilos de aprendizagem. 
Rosário (2006) utilizou o ILS com 257 estudantes de Engenharia Química e de Engenharia de Alimentos; os resultados indicaram que os homens tiveram mais preferência pelo estilo visual enquanto as mulheres apresentaram preferência pelo estilo Verbal.

$\mathrm{Na}$ pesquisa realizada por Belhot et al. (2005) com 123 alunos de Engenharia as mulheres tendem a ser mais sequenciais e mais verbais que os homens. No entanto, não houve diferenças significativas em relação à variável gênero no estudo de Figueiredo e colaboradores (2008).

Trabalhos que utilizaram outros instrumentos para avaliação de estilos de aprendizagem entre estudantes de Farmácia, ora encontraram, ora não encontraram diferenças entre gêneros (Adamcik et al, 1996; Carroll \& Garavalia, 2002).

\section{Conclusão}

A análise dos dados do inventário LASSI no presente estudo aponta para um perfil dos alunos de Farmácia com preferências pelas seguintes estratégias de aprendizagem: preocupações ao estudar, processamento da informação e auxiliares de estudo.

Não foi verificada associação entre as variáveis sociodemográficas e socioeducacionais e as preferências por estratégias de aprendizagem na amostra de alunos que responderam o instrumento LASSI.

Considerando diferentes períodos do curso foram encontradas diferenças significativas na utilização das estratégias de estudo e aprendizagem referentes à subescala de concentração e utilização de auxiliares de estudo. Não houve diferenças significativas nas outras subescalas de estratégias de aprendizagem, o que levanta a hipótese de que o ambiente acadêmico e as relações de ensino-aprendizagem no decorrer do Curso de graduação em Farmácia da UFS pouco interferiram no desenvolvimento daquelas estratégias.

Os resultados dos dados do ILS observados no presente estudo indicaram que há praticamente um equilíbrio no perfil de preferências de estilo de aprendizagem ativo/reflexivo, enquanto há moderada preferência do estilo visual sobre o verbal e do estilo sensorial sobre o intuitivo. Apesar destas considerações, o grupo de estudantes pode ser caracterizado com o seguinte perfil predominante de estilos (segundo o modelo ILS): sensorial, visual, ativo e sequencial.

Não foi evidenciada nenhuma associação estatisticamente relevante entre as variáveis sociodemográficas e socioeducacionais e os estilos de aprendizagem. Da mesma forma, a aplicação de tratamento estatístico não demonstrou qualquer associação entre o período de curso e a preferência por algum estilo de aprendizagem.

Apesar desses resultados, a literatura em pesquisa educacional de cunho sociointeracionista, bem como estudos na área das ciências da cognição aponta que fatores como a interferência do ambiente social e de estudo devem ser investigados para avaliar o grau de influência dos processos de ensino-aprendizagem e recomenda-se que sejam levados em consideração no momento das intervenções pedagógicas.

\section{Referências}

Adamcik, B. A. (1996). Assessment of Pharmacy Students' Critical Thinking and Problem-Solving Abilities. The American Journal of Pharmaceutical Education, 60.

Barreto, I. D. P., Gomes, P. A., Furlaneto, I. P., \& Barreto, B. (2019). Avaliação das estratégias de autoaprendizagem em alunos de um curso de medicina em Belém-Pará. Revista Brasileira de Educação Médica, 43, 36-46.

Bartalo, L., \& Guimarães, S. É. R. (2008). Estratégias de estudo e aprendizagem de alunos universitários: um estudo exploratório. Informação \& Informação, $13(2), 1-14$

Belhot, R. V. (1997). Reflexões e propostas sobre o "ensinar engenharia” para o século XXI. Escola de Engenharia de São Carlos, Universidade de São Paulo, São Carlos.

Belhot, R. V., Freitas, A. A., \& Dornellas, D. V. (2005). Benefícios do conhecimento dos estilos de aprendizagem no ensino de engenharia de produção. In XXXIII Congresso Brasileiro de Educação em Engenharia, COBENGE, Campina Grande-PB. 
Boruchovitch, E., Góes, N. M., Acee, T. W., \& Felicori, C. M. (2020). Brazilian students' learning and study strategies in teacher education programs. Educação: teoria e prática.

BRASIL. (2002) Ministério da Educação. Diretrizes Curriculares Nacionais para os Cursos de Graduação em Farmácia. CNE. Resolução CNE/CES 2/2002. Diário Oficial da União, Brasília, 4 de março de 2002. Seção 1, p. 9

BRASIL. (2017) Ministério da Educação. Diretrizes Curriculares Nacionais para os Cursos de Graduação em Farmácia. Resolução CNE/CES 6/2017. Diário Oficial da União, Brasília, 20 de outubro de 2017, Seção 1, p. 30.

Carroll, C. A., \& Garavalia, L. S. (2002). Gender and racial differences in select determinants of student success. American Journal of Pharmaceutical Education, 66(4), 382-387.

Carroll, C. A., \& Garavalia, L. S. (2004). Factors Contributing to the Academic Achievement of Pharmacy Students: Use of the Goal-Efficacy Framework. American Journal of Pharmaceutical Education, 68(4).

Childs-Kean, L., Edwards, M., \& Smith, M. D. (2020). A Systematic Review of Learning Style Framework Use in Health Sciences Education. American Journal of Pharmaceutical Education.

Colthorpe, K., Ogiji, J., Ainscough, L., Zimbardi, K., \& Anderson, S. (2017). Metacognitive assessment tasks provide insight into undergraduate pharmacy students"e self-regulated learning behavior. American Journal of Pharmaceutical Education.

Czepula, A. I., Bottacin, W. E., Hipólito Jr, E., Baptista, D. R., Pontarolo, R., \& Correr, C. J. (2016). Predominant learning styles among pharmacy students at the Federal University of Paraná, Brazil. Pharmacy Practice (Granada), 14(1), 0-0.

Felder, R. M. (1993). Reaching the second tier. Journal of college science teaching, 23(5), 286-290.

Felder, R. M., \& Silverman, L. K. (1988). Learning and teaching styles in engineering education. Engineering education, 78(7), 674-681.

Felder, R. M., \& Spurlin, J. (2005). Applications, reliability and validity of the index of learning styles. International journal of engineering education, 21(1), 103-112.

Figueiredo, R. S., Noronha, C. M. S., \& de Oliveira Neto, O. J. (2008). Estilos de aprendizagem no ensino técnico agropecuário das escolas técnicas federais do Estado de Goiás. Revista brasileira de gestão e desenvolvimento regional, 4(2).

Garavalia, L. S., Scheuer, D. A., \& Carroll, C. A. (2002). Comparative analysis of first-and third-year pharmacy students' perceptions of student-regulated learning strategies and motivation. American Journal of Pharmaceutical Education, 66(3), 219-222.

Garvey, M. L. (1983). An assessment of learning styles among pharmacy students.

Mainemelis, C., Boyatzis, R. E., \& Kolb, D. A. (2002). Learning styles and adaptive flexibility: Testing experiential learning theory. Management learning, $33(1), 5-33$

McKeirnan, K. C., Colorafi, K., Kim, A. P., Stewart, A. S., Remsberg, C. M., Vu, M., \& Bray, B. S. (2020). Student Study Behaviors associated with Academic Success in an Active Classroom Pharmacy Curriculum. American Journal of Pharmaceutical Education.

Nisly, S. A., Sebaaly, J., Fillius, A. G., Haltom, W. R., \& Dinkins, M. M. (2020). Changes in Pharmacy Students' Metacognition Through Self-Evaluation During Advanced Pharmacy Practice Experiences. American journal of pharmaceutical education, 84(1).

Pungente, M. D., Wasan, K. M., \& Moffett, C. (2003). Using learning styles to evaluate first-year pharmacy students' preferences toward different activities associated with the problem-based learning approach. American Journal of Pharmaceutical Education, 66, 119.

Rosário, J. D. A. D. (2006). Estilos de aprendizagem de alunos de engenharia Química e engenharia de alimentos da UFSC: o caso da disciplina de Análise e Simulação de Processos.

Soloman, B. A., \& Felder, R. M. (2005). Index of learning styles questionnaire. NC State University. Available online at: http://www. engr. ncsu. edu/learningstyles/ilsweb. html (last visited on 14.05. 2010), 70.

Teevan, C. J., Li, M., \& Schlesselman, L. S. (2011). Index of learning styles in a US school of pharmacy. Pharmacy Practice, 9(2), 82.

Yamamura, S., \& Takehira, R. (2018). An Analysis of the Relationship between the Learning Process and Learning Motivation Profiles of Japanese Pharmacy Students Using Structural Equation Modeling. Pharmacy (Basel, Switzerland), 6(2), 35. 